\section{PerFRACTION ${ }^{T M}$}

3D Pre-Treatment QA and In-Vivo Monitoring

- Fraction $0^{\mathrm{TM}}$ Pre-treatment phantom-free QA with the independence and peace of mind of EPID measurements

- Fraction $n^{\mathrm{TM}}$ In-vivo QA for every treatment catches patient and machine errors

- See which clinical goals pass and fail for each daily fraction

- Automated DICOM file capture, analysis, and email notifications for failure

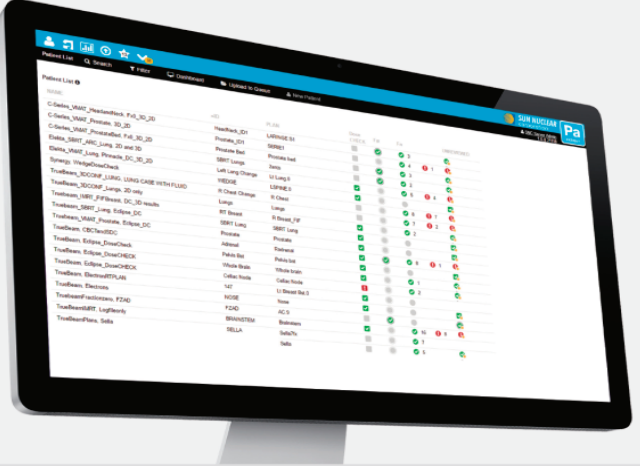

\section{Stereotactic QA}

New Solutions

\section{SRS MapCHECK}

Small Field Digital Film Replacement for Patient Specific QA \& End-to-End Testing

- Designed to insert into StereoPHAN ${ }^{m}$ and run on SNC Patient ${ }^{\text {tw }}$ software

Detector Spacing: $2.47 \mathrm{~mm}$; Detector Resolution: $0.47 \mathrm{~mm}$ diameter

- Measures field sizes as small as $5 \mathrm{~mm}$; 5 diodes in $5 \mathrm{~mm}$ cone

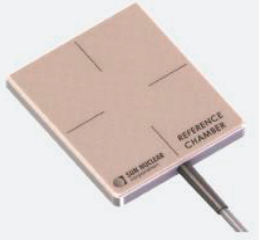

Reference Detector

An Out-of-Field Reference Detector for Water Tank Scanning

Measures LINAC head leakage, enabled by large-volume ion chamber

Used during photon beam commissioning measurements of any field size

Eliminates scanning chamber interference in small SRS fields

- Volume: 39cc; 2-meter cable with triaxial connector
DoseCHECK ${ }^{\mathrm{TM}}$

Independent Secondary 3D Dose Calculations

- Automated, TPS grade dose volume generation

- Efficient dose-to-dose evaluation

- Seamless integration with the PerFRACTION ${ }^{\mathrm{TM}}$ solution for patient QA

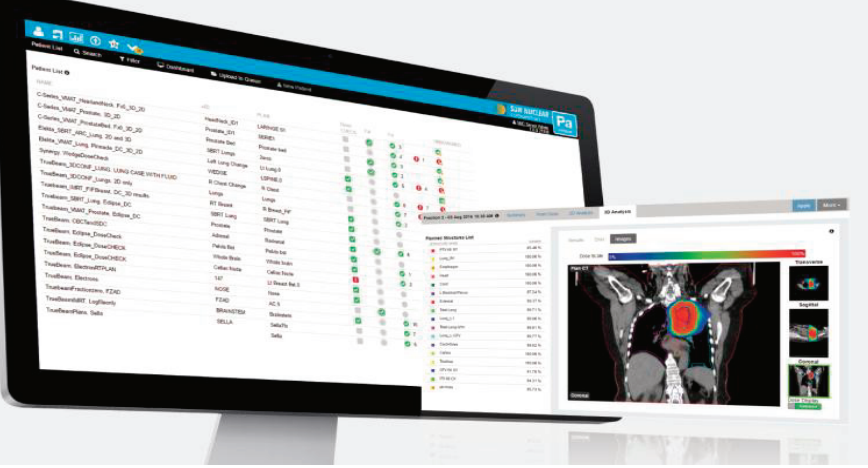

SNC Machine ${ }^{\mathrm{TM}}$

TG-142/VMAT Imaging and Mechanical QA

- Automated workflow captures and analyzes files - no further work required

- Supports most common mechanical and imaging phantoms

- Trend any test parameter against any other test parameter for any number of machines

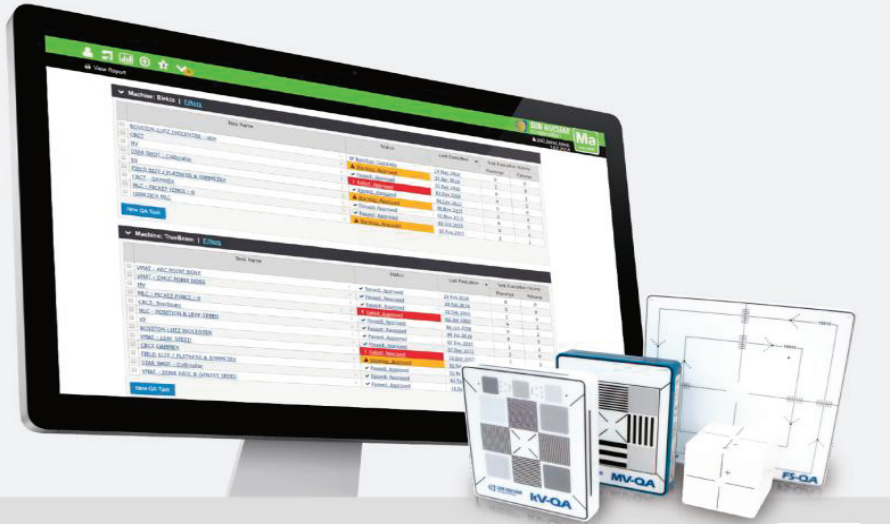




\title{
Design of Multipurpose Phantom for External Audit on Radiotherapy
}

\author{
Sangwook Lim ${ }^{(1)}$ \\ Department of Radiation Oncology, Kosin University College of Medicine, Busan, Korea
}

\author{
Received 22 October 2021 \\ Revised 6 December 2021 \\ Accepted 8 December 2021 \\ Corresponding author \\ Sangwook Lim \\ (medicalphysics@hotmail.com) \\ Tel: 82-51-990-6393
}

Purpose: This study aimed to design a multipurpose dose verification phantom for external audits to secure safe and optimal radiation therapy.

Methods: In this study, we used International Atomic Energy Agency (IAEA) LiF powder thermoluminescence dosimeter (TLD), which is generally used in the therapeutic radiation dose assurance project. The newly designed multipurpose phantom (MPP) consists of a container filled with water, a TLD holder, and two water-pressing covers. The size of the phantom was designed to be sufficient $\left(30 \times 30 \times 30 \mathrm{~cm}^{3}\right)$. The water container was filled with water and pressed with the cover for normal incidence to be fixed. The surface of the MPP was devised to maintain the same distance from the source at all times, even in the case of oblique incidence regardless of the water level. The MPP was irradiated with 6, 10, and 15 MV photon beams from Varian Linear Accelerator and measured by a $1.25 \mathrm{~cm}^{3}$ ionization chamber to get the correction factors. Monte Carlo (MC) simulation was also used to compare the measurements.

Results: The result obtained by MC had a relatively high uncertainty of $1 \%$ at the dosimetry point, but it showed a correction factor value of $1.3 \%$ at the $5 \mathrm{~cm}$ point. The energy dependence was large at $6 \mathrm{MV}$ and small at $15 \mathrm{MV}$. Various dosimetric parameters for external audits can be performed within an hour.

Conclusions: The results allow an objective comparison of the quality assurance (QA) of individual hospitals. Therefore, this can be employed for external audits or QA systems in radiation therapy institutions.

Keywords: External audit, Radiation therapy, Phantom, Photon, Thermoluminescence dosimeter

\section{Introduction}

It is fundamental to ensure the accuracy of the output of the treatment device and the basic quality of the radiation used for treatment, and the treatment method implemented by each treatment institution must be considered. Therefore, it is necessary to prepare a program for securing clinical dose that reflects the high-quality treatment methods implemented in domestic medical institutions.

External audit on radiotherapy program is settled in many countries to increase radiotherapy quality. It can be performed by visiting [1-4] participant centers or by mailing [5-11]. Various international organizations, such as the International Atomic Energy Agency (IAEA), Radiological Physics Center, and ESTRO-QUALity assurance network (EQUAL), operate mailed dosimetry services [12-14].

The goal of these programs was to check only the output parameters of radiotherapy units. However, accuracy is needed in radiotherapy, and complexity is growing in the treatment process $[15,16]$. Therefore, the requirement for an 
effective quality assurance (QA) program has to be emphasized.

The concept of the multipurpose phantom (MPP) designed in this study is to measure various parameters at once for complex radiotherapy audit.

\section{Materials and Methods}

\section{Parameters to be measured}

Referring to the recommendation of ESTRO, AAPM, and routine QA parameters of hospitals, the essential parameters were chosen for radiotherapy audit [15]. The parameters were for 4 to $20 \mathrm{MV}$ photon beams. The MPP was constructed to check on- and off-axis in three irradiation conditions in a vertical and oblique beam setup, as shown in Table 1.

Table 1. Beam setup and parameters to be measured

\begin{tabular}{cl}
\hline \multicolumn{1}{c}{ Beam setup } & Parameter \\
\hline Square field & Output \\
$-10 \times 10 \mathrm{~cm}^{2}$ of field size & Symmetry \\
- Reference depth of $5 \mathrm{~cm}$ & Flatness \\
& PDD \\
& Inhomogeneity \\
Oblique field & Profile \\
$-10 \times 10 \mathrm{~cm}^{2}$ of field size & PDD \\
$-30^{\circ}$ rotation of gantry & \\
Wedged field & Wedge factor \\
$-30^{\circ}$ wedge & Profile \\
$-10 \times 10 \mathrm{~cm}^{2}$ of field size & PDD \\
$-30^{\circ}$ rotation of gantry &
\end{tabular}

PDD, percent depth dose.

\section{Requirements}

The phantom was developed under the following conditions. First, the phantom should be water equivalent materials. Second, it should be possible to measure multiple factors at once. Third, all measurements must be completed within an hour. Fourth, it should be simple to set up and easy to use. Fifth, it should be light in weight and convenient to deliver. Sixth, it should be possible to use the thermoluminescence dosimeter (TLD) previously used by IAEA for postal audit.

\section{Thermoluminescence dosimeter}

The TLDs used in the IAEA QA program were capsules filled with approximately $155 \mathrm{mg}$ of $\mathrm{LiF}$ powder. The capsule was a black polyethylene cylinder of $20 \mathrm{~mm}$ inner length, 3 $\mathrm{mm}$ inner diameter, and $1 \mathrm{~mm}$ wall thickness (Fig. 1) [13]. The MPP designed in this study used 7 or 9 TLDs at a time.

\section{Design of multipurpose phantom}

The MPP was composed of (a) a water container, (b) a TLD holder, (c) a cover for normal incidence, and (d) a cover for oblique incidence, as shown in Fig. 2. The water container and support rod were made of acrylic, which has a density similar to that of water, and were designed to be filled with water in use to reduce weight during delivery. The AAPM TG-51 protocol recommended the use of water [17]. The size of the phantom was designed to be sufficient $\left(30 \times 30 \times 30 \mathrm{~cm}^{3}\right)$, considering the back and side scatters. The TLD holder was designed to be inserted into the water phantom after the TLDs were applied, making it easy to
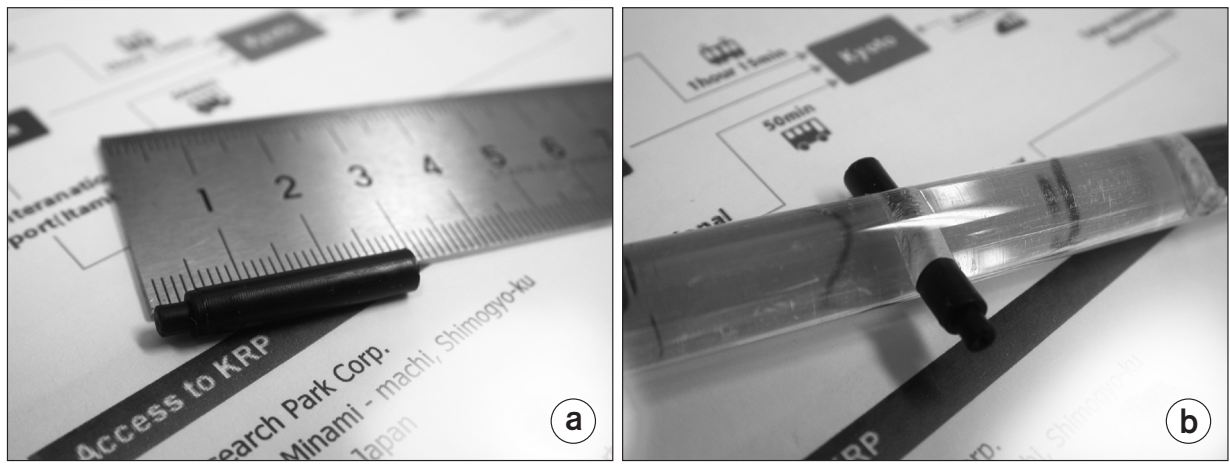

Fig. 1. IAEA capsulated $155 \mathrm{mg}$ of $\mathrm{LiF}$ powder TLD (a) the capsule measures $20 \mathrm{~mm}$ inner length, $3 \mathrm{~mm}$ inner diameter, and $1 \mathrm{~mm}$ wall thickness and (b) TLD in TLD holder. IAEA, International Atomic Energy Agency; TLD, thermoluminescence dosimeter. 

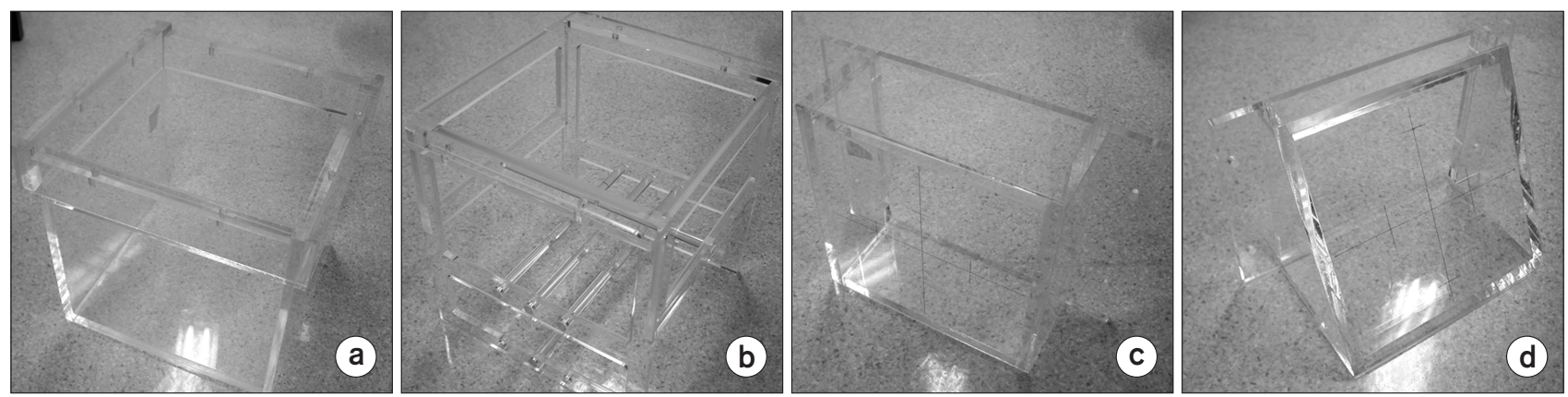

Fig. 2. Component of the multipurpose phantom. (a) Water container. (b) Thermoluminescence dosimeter holder. (c) Cover for normal incident. (d) Cover for oblique incidence.
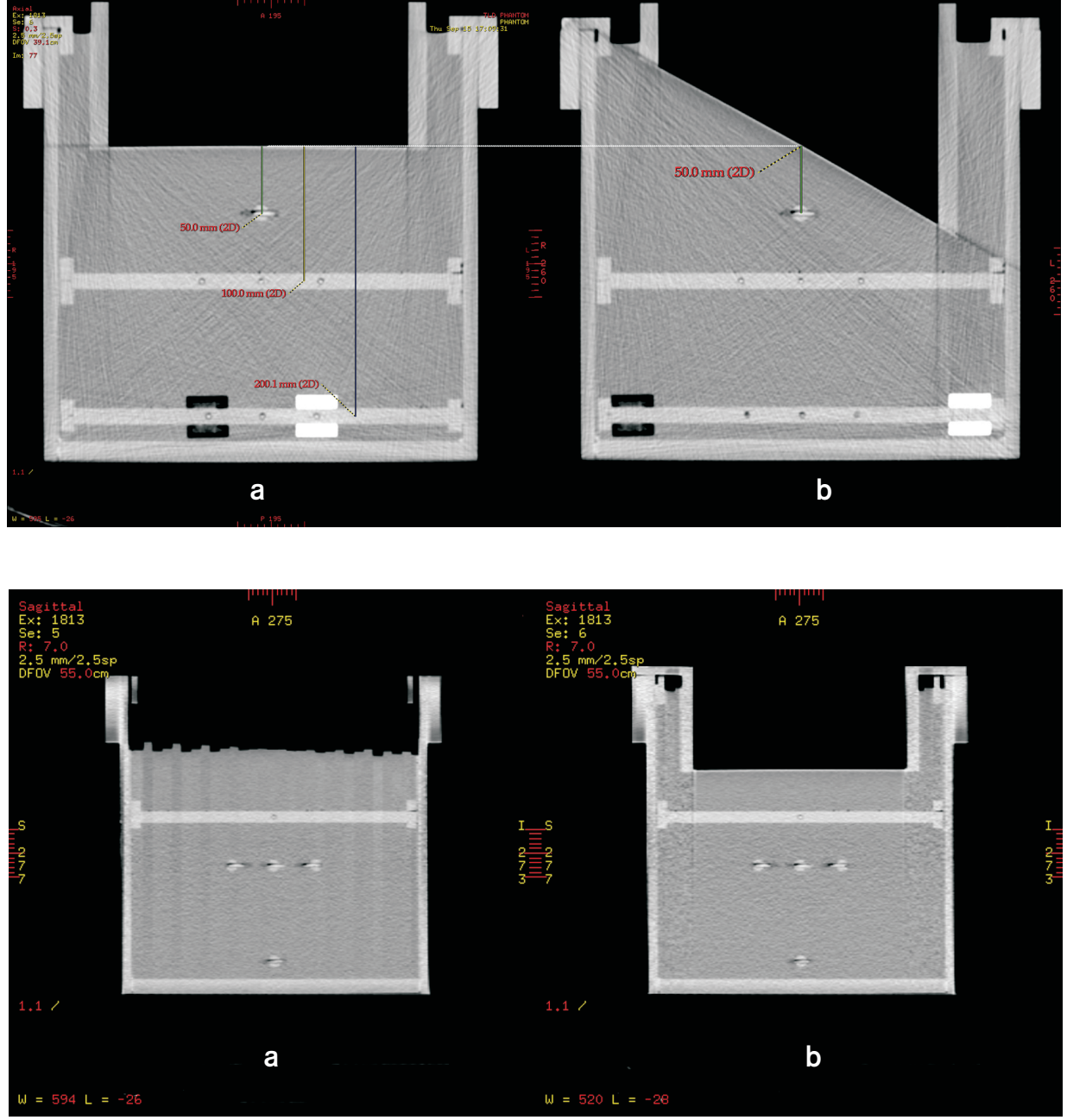

Fig. 3. Water-pressing cover can be selected for (a) normal incidence setup and (b) oblique incidence setup. Regardless of the water level, the source to surface distance is always constant because of the water-pressing cover. Since the two covers are designed to have the same volume when submerged in water, the water level is the same.
Fig. 4. The multipurpose phantom was designed to allow computed tomography (CT) scans. (a) Sagittal CT image without pressing cover: moving couch-induced slopping of water during helical CT scan causes beat phenomenon in CT image. (b) Sagittal CT image water-pressing cover: waterpressing cover prevents the water from slopping during helical CT scan. replace the TLD during measurement. In this study, the ionization chamber can be inserted at the same locations as the TLDs to evaluate the reliability of the MPP. The water container was filled with water and pressed with the cover for normal incidence to be fixed. The surface of the MPP was devised to maintain the same distance from the source at all times, even in the case of oblique incidence regardless of the water level (Fig. 3). Additionally, the covers prevented the water from sloshing due to the movement of the computed tomography (CT) table during the CT scan, and there was no sloshing artifact (Fig. 4). To minimize the attenuation of photon beams, the thickness of the acrylic window 
of the lid was designed to be the minimum thickness (2 $\mathrm{mm}$ ) that can withstand the pressure of water.

\section{Multipurpose phantom setup and measurement}

The locations of the TLDs in the phantom are shown in Fig. 5, where one TLD is located at a depth of $5 \mathrm{~cm}$ from the surface, and 5 TLDs are located at a depth of $10 \mathrm{~cm}$ to determine the dose, flatness, and symmetry. At a depth of $20 \mathrm{~cm}$, one TLD was used for depth dose, and two TLDs were for heterogeneity measurement. The dimensions of inhomogeneous materials were $3 \times 3 \times 3 \mathrm{~cm}^{3}$, Teflon $\left(2.35 \mathrm{~g} / \mathrm{cm}^{3}\right)$, which has a density similar to that of human bone, and cork $(0.32$ $\mathrm{g} / \mathrm{cm}^{3}$ ), which has a density similar to the lungs, were used, and the TLDs were designed to be inserted. The density of thermoluminescence was $2.65 \mathrm{~g} / \mathrm{cm}^{3}$, equivalent to $8 \mathrm{~mm}$ depth of water.

When not in use, the two inhomogeneous materials can be pushed to either end. The total number of TLDs required for all measurements was 23 per energy.

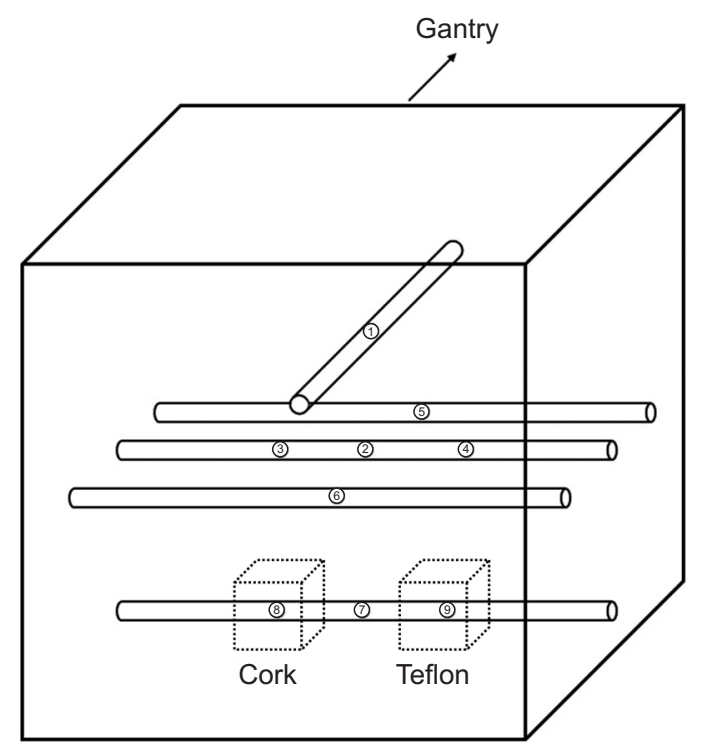

Fig. 5. Location of TLDs in multipurpose phantom. The numbers from (1) to (9) indicate the location of the TLD. Location of (1) is at $5 \mathrm{~cm}$ depth, location of (2)-(6) is at $10 \mathrm{~cm}$ depth, and location of (7)-(9) is at $20 \mathrm{~cm}$ depth, and location of (8) and (9) is used for inhomogeneity. TLD, thermoluminescence dosimeter.

\section{Correction factors for multipurpose phantom and water phantom}

The first method used to evaluate the perturbation at each point in an MPP was a measurement using an ionization chamber in the reference water phantom used to obtain beam data in a treatment institution.

The MPP was irradiated with 6, 10, and 15 MV photon beams from Varian Linear Accelerator and measured by a $1.25 \mathrm{~cm}^{3}$ ionization chamber (PTW) to evaluate the MPP. All energies were measured by an ionization chamber in the same geometric setup as the TLD to compare it with TLD measurements.

First, the doses were measured using the ionization chamber installed in a reference water phantom at the same location where the TLDs were installed in the MPP. Next, the dose was measured using the ionization chamber in 9 locations in the MPP.

To convert the doses measured in the MPP into the dose value measured in the water phantom, the following dose correction factors are defined as follows:

$$
C F=D_{M P P} / D_{W P}
$$

where $C F$ is the correction factor, $D_{M P P}$ is the dose measured by the ionization chamber in MPP, and $D_{W P}$ is the dose measured by the ionization chamber in the reference water phantom.

If the dose measured using the TLD in the MPP is $D_{M P P}$, the dose in the water phantom, $D_{W P}=D_{M P P} / C F$, can be obtained.

\section{Correction factor for thermoluminescence dosimeter vs. Monte Carlo (MC) simulation}

The NRC (National Research Council Canada) has calculated and announced photon spectra from 9 machines. The spectrum presented by the NRC for Varian 6, 10, and $15 \mathrm{MV}$ and Siemens 6 and 10 MV was used in this study.

The MPP was modeled as shown in Fig. 6, and all points were modeled assuming water. MC simulation was performed in Monte Carlo N-Particle Transport Code Systemextended (MCNPX, version 2.4.0), photons and electrons 


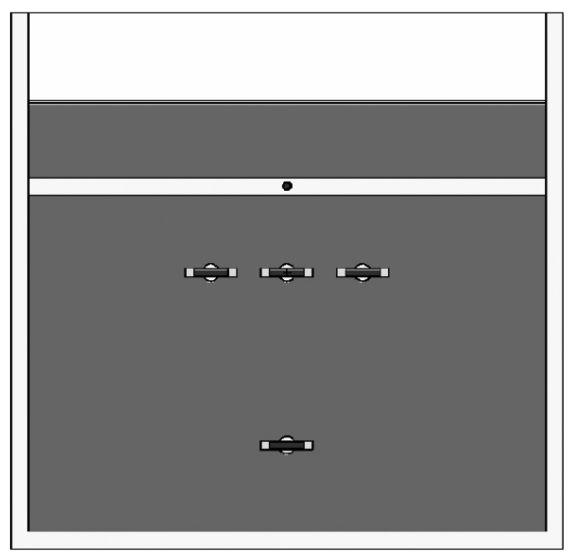

a

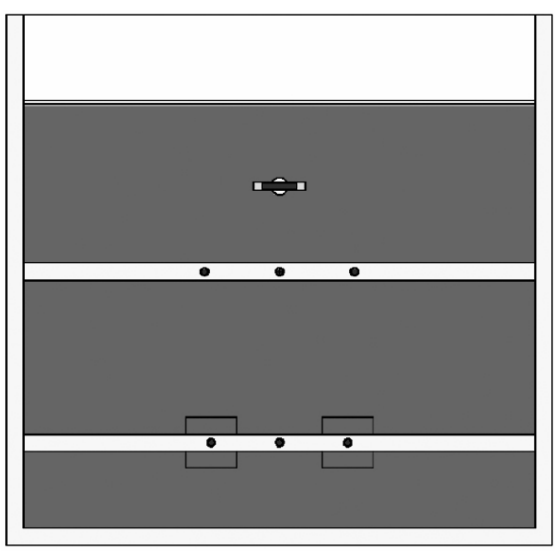

b
Fig. 6. Modeling of multipurpose phantom using MCNPX. (a) Front view. (b) Lateral view.

Table 2. Correction factors obtained from measurements of ionization chamber in MPP and water phantom for normal incidence

\begin{tabular}{ccccc}
\hline \multirow{2}{*}{ Depth $(\mathrm{cm})$} & $\begin{array}{c}\text { Location } \\
\text { number }\end{array}$ & $6 \mathrm{MV}$ & Correction factor (MPP/water phantom) & $15 \mathrm{MV}$ \\
\cline { 2 - 4 } 5 & 1 & 0.9888 & 0.9765 & 0.9873 \\
10 & 2 & 0.9814 & 0.9715 & 0.9911 \\
& 3 & 0.9925 & 0.9746 & 0.9960 \\
& 4 & 0.9896 & 0.9823 & 0.9853 \\
& 5 & 0.9883 & 0.9754 & 0.9828 \\
& 6 & 0.9767 & 0.9909 & 0.9799 \\
& 7 & 0.9822 & 0.9828 & 0.9914 \\
& 8 & 0.9895 & 0.9815 & 0.9915 \\
& 9 & 0.9860 & 0.9854 & 0.9885 \\
\hline
\end{tabular}

MPP, multipurpose phantom.

were traced to $1 \mathrm{keV}$ and $100 \mathrm{keV}$, respectively, and F8 tally, a pulse height tally, was used to calculate energy absorption. In the case of the F8 tally, it can be used as a direct energy absorption because the energy transferred within the volume of the measurement point can be tracked by tracking the movement of particles.

\section{Results}

\section{Results of correction factor for multipurpose phantom and water phantom}

Fig. 6 shows the doses measured in the water phantom and the MPP at 9 points, and Table 2 shows the correction factors obtained using the above equation. The geometrical positions of the ionization chamber in the MPP and the 3D water phantom are the same.

Fig. 7a shows the measured value in the case of normal incident, Fig. 7b shows the measured value in the case of oblique incident, and Fig. 7c shows the measured value in the case of oblique incident with a $30^{\circ}$ wedge. In Fig. $7 \mathrm{~b}$, positions 2 to 6 are measured values at a depth of $10 \mathrm{~cm}$.

The correction factors obtained from MC simulations are shown in Table 3 and plotted in Fig. 8.

\section{Uncertainty of multipurpose phantom for setup and energies}

The Type A standard uncertainties were taken from the deviation of TLD measurements based on ionization chamber measurements in MPP. The measurements were repeated 18 for normal and 14 for oblique and wedged incidences. The Type A standard uncertainties of the MPP for each energy and setup are shown in Table 4. The Type A standard uncertainties were calculated as follows: 
a
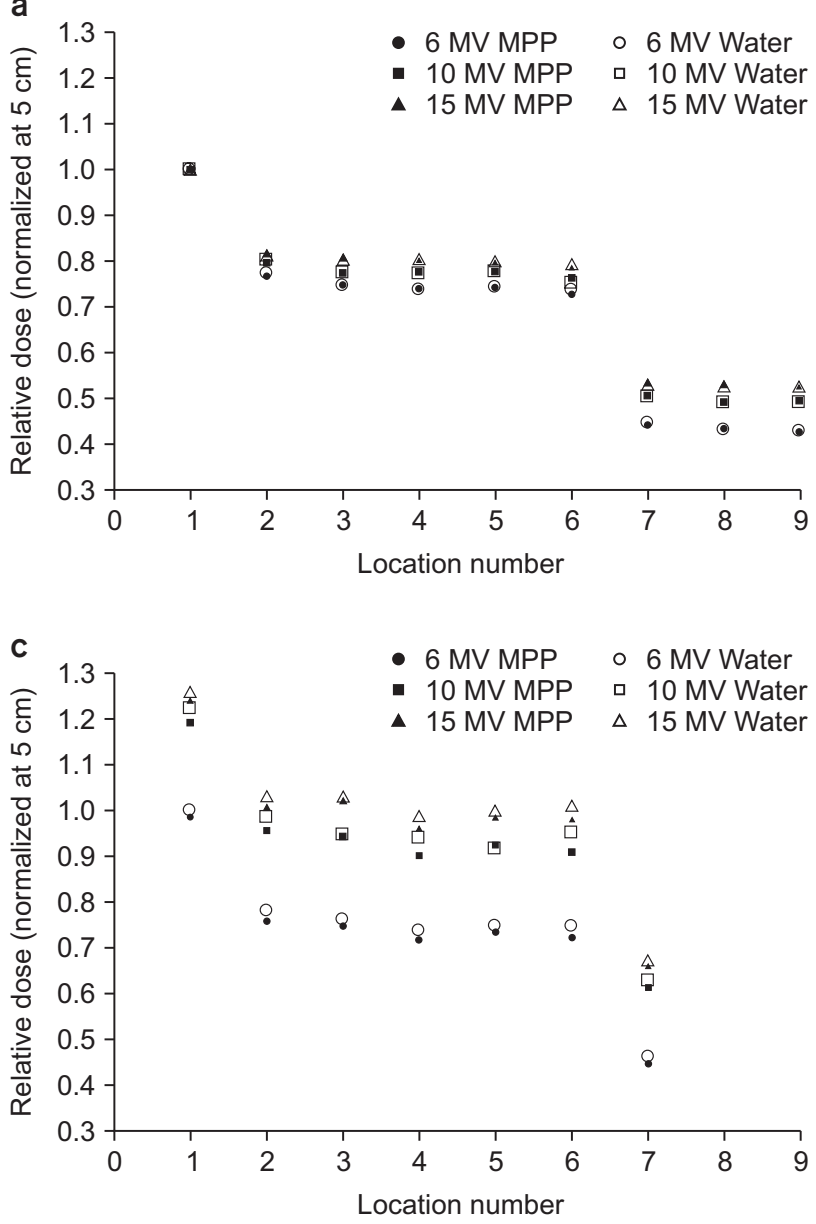

b

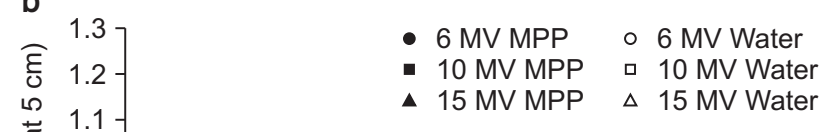

- 10 MV Water

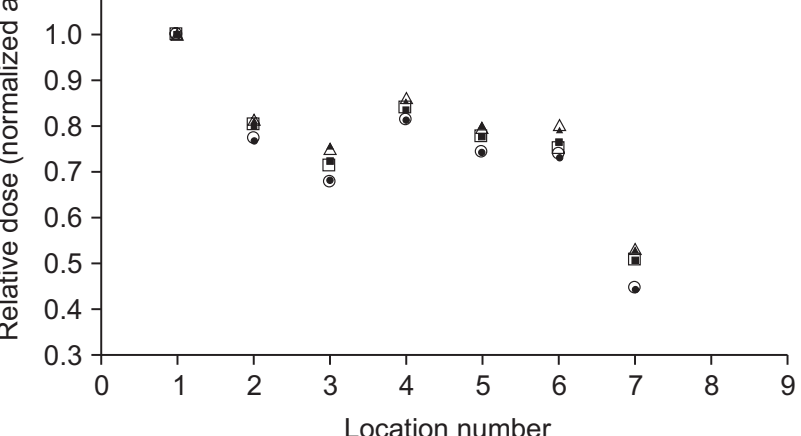

Fig. 7. Relative dose of each point measured by ionization chamber. (a) Relative dose for normal incident. (b) Relative dose for oblique incident. (c) Relative dose for oblique incident with a $30^{\circ}$ wedge. MPP, multipurpose phantom.

Table 3. Correction factors calculated by MCNPX 2.4.0 at each point

\begin{tabular}{cccccc}
\hline $\begin{array}{c}\text { Location } \\
\text { number }\end{array}$ & Varian 6 MV & Siemens 6 MV & Siemens 10 MV & Varian 10 MV & Varian 15 MV \\
\hline 1 & 0.9875 & 0.9874 & 0.9897 & 0.9828 & 0.9904 \\
2 & 0.9693 & 0.9702 & 0.9846 & 0.9790 & 0.9779 \\
3 & 0.9793 & 0.9783 & 0.9867 & 0.9859 & 0.9807 \\
4 & 0.9875 & 0.9912 & 0.9864 & 0.9809 & 0.9711 \\
5 & 0.9817 & 0.9697 & 0.9768 & 0.9730 & 0.9729 \\
6 & 0.9817 & 0.9683 & 0.9806 & 0.9663 & 0.9683 \\
7 & 0.9653 & 0.9679 & 0.9740 & 0.9863 & 0.9785 \\
9 & 0.9825 & 0.9796 & 0.9835 & 0.9943 & 0.9878 \\
\hline
\end{tabular}

Uncertainty $=\frac{S D}{\sqrt{N}}$

where $\mathrm{SD}$ is the standard deviation of the sample and $\mathrm{N}$ is the number of samples.

\section{Discussion}

Since the values of positions 2 to 6 in Fig. $7 \mathrm{~b}$ were measured for oblique incidents, they differ depending on the depth reached. However, in Fig. 7c, since the wedge is compensated, it can be seen that the values are almost the same 


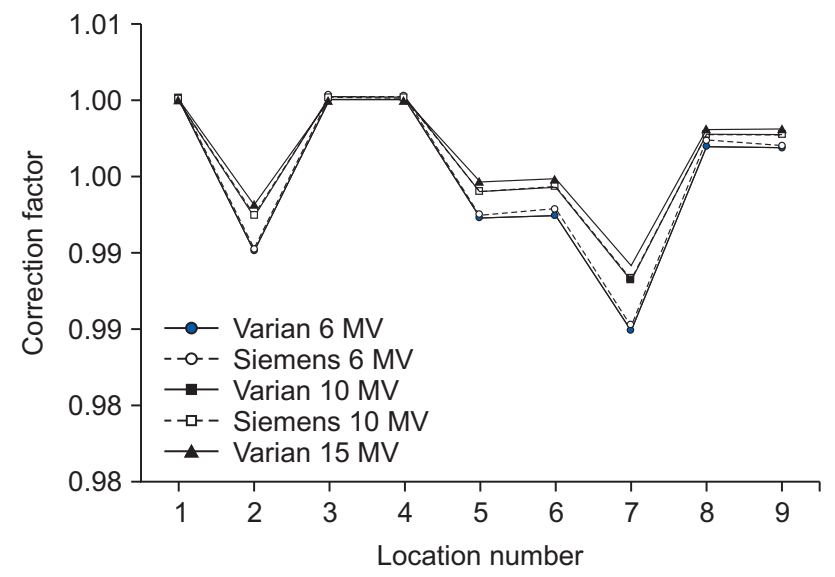

Fig. 8. Correction factors plotted for each machine and energy.

as in Fig. 7a.

The result obtained by MC had a relatively high uncertainty of $1 \%$ at the dosimetry point, and it showed a correction factor value of $1.3 \%$ at the $5 \mathrm{~cm}$ point. This result was similar to that of Mobit et al.'s experiment [18], which agrees within $1.5 \%$ between measurements and MC calculations.

The energy dependence was large at $6 \mathrm{MV}$ and small at 15 MV. This is thought to be because the higher the energy of the photon beam, the stronger the penetrating power.

\section{Conclusions}

With the MPP developed in this study, the measurement of various dosimetric parameters for external audit can be performed within an hour.

The MPP is not limited to measuring dosimetric data in perpendicular incidence to the water surface but performs additional measurements at oblique incidence and close to clinical cases such as flatness, symmetry, oblique beam, and inhomogeneity without gantry rotations.

According to MC calculation and ionization chamber measurements, the energy correction factor should be considered.

The MPP has been demonstrated to be useful in accessing the overall accuracy of the treatment units by dosimetric checking of photon beams in reference and nonreference conditions and in checking the TPS by verifying the calculated data with the measured data. The MPP developed in this study is considered useful for external audits or QA sys-
Table 4. Type A standard uncertainties according to setup and energies

\begin{tabular}{lccc}
\hline Energy & Normal (\%) & Oblique (\%) & Wedged (\%) \\
\hline $6 \mathrm{MV}$ & 0.54 & 0.66 & 0.43 \\
$10 \mathrm{MV}$ & 0.48 & 0.69 & 0.43 \\
$15 \mathrm{MV}$ & 0.54 & 0.66 & 0.43 \\
\hline
\end{tabular}

tems of radiation therapy institutions.

\section{Conflicts of Interest}

The authors have nothing to disclose.

\section{Availability of Data and Materials}

All relevant data are within the paper and its Supporting Information files.

\section{References}

1. Hoornaert MT, Van Dam J, Vynckier S, Bouiller A. A dosimetric quality audit of photon beams by the Belgian Hospital Physicist Association. Radiother Oncol. 1993;28:37-43.

2. Nisbet A, Thwaites DI, Sheridan ME. A dosimetric intercomparison of kilovoltage X-rays, megavoltage photons and electrons in the Republic of Ireland. Radiother Oncol. 1998;48:95-101.

3. Nisbet A, Thwaites DI. A dosimetric intercomparison of electron beams in UK radiotherapy centres. Phys Med Biol. 1997;42:2393-2409.

4. Choi CH, Kim JI, Park JM, Park YK, Cho KW, Cho WK, et al. External auditing on absorbed dose using a solid water phantom for domestic radiotherapy facilities. J Korean Soc Ther Radiol Oncol. 2010;28:50-56.

5. Davis B, Faessler P. Quality audit of megavoltage radiotherapy units: intercomparison of dose at a reference point using a mailed TL-dosimetry system. Radiother Oncol. 1993; 28:79-81.

6. Derreumaux S, Chavaudra J, Bridier A, Rossetti V, Dutreix A. A European quality assurance network for radiotherapy: dose measurement procedure. Phys Med Biol. 1995;40:11911208.

7. Dutreix A, Derreumaux S, Chavaudra J, van der Schueren 
E. Quality control of radiotherapy centres in Europe: beam calibration. Radiother Oncol. 1994;32:256-264.

8. Dutreix A, van der Schueren E, Derreumaux S, Chavaudra J. Preliminary results of a quality assurance network for radiotherapy centres in Europe. Radiother Oncol. 1993;29:97101.

9. Hansson U, Johansson KA. Quality audit of radiotherapy with EORTC mailed in water TL-dosimetry. Radiother Oncol. 1991;20:191-196.

10. Huntley R, Izewska J. The 1998 Australian external beam radiotherapy survey and IAEA/WHO TLD postal dose quality audit. Australas Phys Eng Sci Med. 2000;23:21-29.

11. Izewska J, Novotny J, Gwiazdowska B, Kindlova A, Kontra G, van Dam J, et al. External audit on output calibration for photon beams. Acta Oncol. 1995;34:829-838.

12. Ferreira IH, Dutreix A, Bridier A, Chavaudra J, Svensson H. The ESTRO-QUALity assurance network (EQUAL). Radiother Oncol. 2000;55:273-284.

13. Izewska J, Andreo P. The IAEA/WHO TLD postal programme for radiotherapy hospitals. Radiother Oncol. 2000; 54:65-72.
14. Svensson H, Hanson GP, Zsdanszky K. SSDL newsletter. No. 28. The IAEA/WHO TL dosimetry service for radiotherapy centres. 1969-1987. Vienna: International Atomic Energy Agency (IAEA); 1989:3-23.

15. Swinnen A, Verstraete J, Huyskens D. The use of a multipurpose phantom for mailed dosimetry checks of therapeutic photon beams: 'OPERA' (operational phantom for external radiotherapy audit). Radiother Oncol. 2002;64:317326.

16. Bridier A, Nyström H, Ferreira I, Gomola I, Huyskens D. A comparative description of three multipurpose phantoms (MPP) for external audits of photon beams in radiotherapy: the water MPP, the Umeå MPP and the EC MPP. Radiother Oncol. 2000;55:285-293.

17. Almond PR, Biggs PJ, Coursey BM, Hanson WF, Huq MS, Nath R, et al. AAPM's TG-51 protocol for clinical reference dosimetry of high-energy photon and electron beams. Med Phys. 1999;26:1847-1870.

18. Mobit PN, Mayles P, Nahum AE. The quality dependence of LiF TLD in megavoltage photon beams: Monte Carlo simulation and experiments. Phys Med Biol. 1996;41:387-398. 